\title{
Aberrant Chromosome 21 Gene Products: Explaining the Down Syndrome Phenotype?
}

\author{
Rosa Ferrando-Miguel Myeong Sook Cheon Gert Lubec \\ Department of Pediatrics, Division of Basic Sciences, University of Vienna, Vienna, Austria
}

Although Down syndrome (DS; trisomy 21) has been known for more than a hundred years, mechanisms to explain the development of the DS phenotype are elusive. Also, sequencing of the entire human chromosome 21 did not lead to a breakthrough. A few candidate genes encoded on chromosome 21 have been proposed to play a pathogenetic role, but most studies were carried out in nonneural tissues and are therefore not useful for explaining the CNS phenotype and mental retardation, the cardinal symptom of DS besides dysmorphic features.

The majority of studies on genetic changes in DS have been carried out at the RNA level, but there is a long and unpredictable pathway from RNA to the proteins that carry out functions, and systematic work on protein expression in the brain is missing. There are, however, several publications on individual protein derangements $[1,2]$, and we therefore decided to determine chromosome 21 gene products in fetal human DS brain.

We used cortical tissue of fetal female controls $(n=6)$ and DS brain $(\mathrm{n}=8)$ from the 18th-19th week of gestation (obtained from the Fetal Down Syndrome Brain Bank, Dr. M. Dierssen, Barcelona, Spain). This tissue was homogenized, then the protein concentration was determined and Western blotting was performed on samples as described previously [3]. The antibodies used were obtained from other scientists or purchased, and corre- sponding secondary antibodies were commercially available. Some proteins were determined by two-dimensional gel electrophoresis with subsequent mass spectrometric identification and quantification using specific software.

Quantification of immunoreactivities showed that chromosome 21 gene products in fetal DS brain were comparable to those of controls for the vast majority of observations. A handful of proteins whose genes are encoded on chromosome 21 were aberrantly expressed. The human reduced folate carrier was overexpressed in DS, and this may be of relevance for abnormal development of the brain in DS, as deterioration of folate metabolism has already been reported in DS and has even been linked to nondisjunction and an increased risk of developing DS [4]. Chromosome assembly factor (CHAF-1 p60) was significantly increased in DS cortex as well, possibly indicating defective DNA repair due to oxidative DNA damage observed in DS. Increased synaptojanin levels in fetal DS brain confirmed previous observations that this protein is manifold (rather than the expected $150 \%$ by gene dosage) upregulated in DS, which may contribute to abnormal signaling in DS brain as this protein represents polyphosphoinositide phosphatase activity. Overexpression of DSCR5, a phosphatidyl-inositol-glycan class P protein, in turn points to the involvement of phosphoinositol cascades in DS.

\section{KARGER \\ Fax + 41613061234 E-Mail karger@karger.ch} www. karger.com (c) 2005 S. Karger AG, Base

$1424-8522 / 05 / 0031-0001 \$ 22.00 / 0$

Accessible online at: www. karger.com/neb
Gert Lubec, CChem, FRSC (UK)

University of Vienna, Department of Pediatrics, Division of Basic Sciences Währinger Gürtel 18

AT-1090 Vienna (Austria)

Tel. +43140400 3215, Fax +43140400 3194, E-Mail gert.lubec@akh-wien.ac.at 
HUMAN FETAL BRAIN PROTEIN EXPRESSION OF GENES ENCODED ON

CHROMOSOME 21

GENES: Black: known genes; Purple: putative genes.

PROTEIN LEVELS: Black: comparable; Red: increased; Blue: decreased; Grey: expression not detectable.

\begin{tabular}{|c|c|c|c|c|c|}
\hline GENES & & PROTEINS & GENES & & PROTEINS \\
\hline CNN2 (CNN2P) & $21 q 11.2$ & Calponin H2 (Q99439) & $\begin{array}{cr}\text { DYRK1A } & 2 \\
\text { (DYRK;HP86;MNB;MNB }\end{array}$ & $\begin{array}{l}\text { 21q22.13 } \\
\text { BH) }\end{array}$ & $\begin{array}{c}\text { Dual-specificity tyrosine- } \\
\text { phosphorylation regulated kinase 1A } \\
(\mathrm{Q13627)})\end{array}$ \\
\hline SAMSN1(HACS1) & $21 q 11.2$ & SAM-domain protein SAMSN-1 (Q9NSI8) & $\begin{array}{c}\text { KCNJ6 } \\
\text { (GIRK2;KCNJ7;KIR3.2;KA }\end{array}$ & $\begin{array}{l}21922.13 \\
\text { ATP2) }\end{array}$ & $\begin{array}{l}\text { G protein-activated inward rectifier } \\
\text { potassium channel } 2(\mathbf{P 4 8 0 5 1 )}\end{array}$ \\
\hline CXADR & $21 q 11.2$ & $\begin{array}{c}\text { Coxsackievirus and adenovirus receptor } \\
\text { [Precursor] (P78310) }\end{array}$ & DSCR4 (DCRB) & 21922.2 & $\begin{array}{c}\text { Down Syndrome critical region } \\
\text { protein } 4 \text { (P56555) }\end{array}$ \\
\hline NCAM2 (NCAM21) & $21 q 22.1$ & $\begin{array}{l}\text { Neural cell adhesion molecule } 2 \text { precursor } \\
\text { (015394) }\end{array}$ & $\begin{array}{c}\text { KCNJ15 } \\
\text { (KCNJ14;Kir4.2;Kir1.3 }\end{array}$ & 3) & $\begin{array}{l}\text { ATP-sensitive inward rectifier } \\
\text { potassium channel } 15 \text { (Q99712) }\end{array}$ \\
\hline APP (A4; AD1;CVAP) & 21921.3 & $\begin{array}{l}\text { Amyloid beta A4 protein [Precursor] } \\
\text { (P05067) }\end{array}$ & ERG (ERG2) & $21 \mathrm{q} 22.2$ & $\begin{array}{c}\text { Transcriptional regulator ERG } \\
\text { (P11308) }\end{array}$ \\
\hline ADAMTS1 (METH1) & 21921.3 & $\begin{array}{c}\text { ADAMTS-1 precursor, } \\
\text { A disintegrin and metalloproteinase with } \\
\text { thrombospondin motifs 1 (Q9UHI8) }\end{array}$ & ETS2 (ETS-2;c-ETS-2 & $\begin{array}{l}-2) \\
21422.2\end{array}$ & C-ets-2 protein (P15036) \\
\hline ADAMTS5 (ADAMTS11) & $21 \mathbf{q 2 1 . 3}$ & $\begin{array}{l}\text { ADAMTS- precursor, Aggrecanase } 2 \\
\text { A disintegrin and metalloproteinase with } \\
\text { thrombospondin motifs } 1 \text { (Q9UNA0) }\end{array}$ & PCP4 (PEP19) & $21 \mathrm{q} 22.2$ & $\begin{array}{l}\text { Brain specific polypeptide PEP-19 } \\
\text { (P48539) }\end{array}$ \\
\hline CCT8 (CCTQ;KIAA0002) & $21 \mathrm{q} 22.11$ & $\begin{array}{l}\text { T-complex protein 1, theta subunit } \\
\text { (P50990) }\end{array}$ & BACE2 (ASP1;CDA13) & $21 \mathrm{q} 22.3$ & Beta secretase 2 precursor (Q14867) \\
\hline BACH1 & $21 \mathrm{q} 22.11$ & $\begin{array}{l}\text { Transcription regulator BACH1, } \\
\text { BTB and CNC homolog } 1 \text { (O14867) }\end{array}$ & MX1 (IFI-78K;IFI78) & 21922.3 & $\begin{array}{c}\text { Interferon-regulated resistance GTP- } \\
\text { binding protein MxA (P20591) }\end{array}$ \\
\hline GRIK1(GLUR5;GLR5) & $21 \mathrm{q22.11}$ & $\begin{array}{c}\text { human glutamate receptor, ionotropic } \\
\text { kainate } 1 \text { (P39086) }\end{array}$ & $\begin{array}{l}\text { ABCG1 (ABC8;WHITE } \\
\text { WHT1; WHITE1-LSB) }\end{array}$ & E; & $\begin{array}{l}\text { ATP-binding cassette, sub-family G, } \\
\text { member } 1 \text { (P45844) }\end{array}$ \\
\hline CLDN8 (Claudin 8) & $21 \mathrm{q} 22.11$ & Claudin-8 (P56748) & TFF3 (HP1.B;ITF;TFI) & 21922.3 & $\begin{array}{l}\text { Trefoil factor } 3 \text { precursor } \\
\text { (Q07654) }\end{array}$ \\
\hline TIAM1 & $21 \mathrm{q} 22.11$ & $\begin{array}{l}\text { T-lymphoma invasion and metastasis } \\
\text { inducing protein } 1 \text { (Q13009) }\end{array}$ & TFF2 (SML1;SP) & $21 \mathrm{q} 22.3$ & Trefoil factor 2 precursor (Q03403) \\
\hline SOD1 (ALS;ALS1) & $21 q 22 . .11$ & Superoxide dismutase $[\mathrm{Cu}-\mathrm{Zn}](\mathrm{P} 00441)$ & TFF1 (BCEI;D21S21;PS2; & $\begin{aligned}\mathrm{HPS} 2) \\
21 \mathrm{q} 22.3 \\
\end{aligned}$ & Trefoil factor 1 precursor (P04155) \\
\hline SYNJ1 & 21922.11 & Synaptojanin $1(043426)$ & PKNOX1 & 21922.3 & $\begin{array}{c}\text { Homeobox protein PKNOX1, } \\
\text { Homeobox protein PREP-1 (P55347) }\end{array}$ \\
\hline OLIGO1 & $21 \mathrm{q} 22.11$ & $\begin{array}{l}\text { Oligodendrocyte transcription factor } 1 \\
\text { (Q8TAK6) }\end{array}$ & CBS & $21 \mathrm{q} 22.3$ & $\begin{array}{l}\text { Cystahionine beta-synthase } \\
\text { (P35520) }\end{array}$ \\
\hline IFNAR2 (IFNARB) & 21922.11 & $\begin{array}{c}\text { Interferon ?/? receptor ? chain [Precursor] } \\
\text { (P48551) }\end{array}$ & CRYAA (CRYA1) & $21 \mathrm{q} 22.3$ & Alpha crystallin A chain (P02489) \\
\hline IFNAR1 (IFNAR;IFRC) 2 & 21 22.11 & $\begin{array}{c}\text { Interferon ?/? receptor ? chain [Precursor] } \\
\text { (P17181) }\end{array}$ & PDXK (PKH;PNK) & $21 \mathrm{q22.3}$ & Pyridoxal kinase (O00764) \\
\hline IFNGR2 (IFGR2;IFNGT1) & $21 \mathrm{q} 22.11$ & $\begin{array}{c}\text { Interferon gamma receptor beta chain } \\
\text { precursor (P38484) }\end{array}$ & CSTB (CST6;STFB) & 21922.3 & Cystatin B (P04080) \\
\hline $\begin{array}{c}\text { GART } \\
\text { (GARS;PGFT;PRGS) }\end{array}$ & 21922.11 & $\begin{array}{c}\text { Trifunctional purine biosynthetic protein } \\
\text { adenosine-3 (P22102) }\end{array}$ & C21orf 33 (ES1; KNPH;HE & \begin{tabular}{|l|l} 
ES1) \\
$21 \mathrm{q} 22.3$ \\
\end{tabular} & $\begin{array}{c}\text { ES1 protein homolog, mitochondrial } \\
\text { precursor (P30042) }\end{array}$ \\
\hline KCNE2 (MirP1) & $21 \mathrm{q22.12}$ & $\begin{array}{c}\text { Potassium voltage-gated channel subfamily } \\
\text { E member } 2 \text { (Q9Y6J6) }\end{array}$ & AIRE & $21 \mathrm{q} 22.3$ & $\begin{array}{c}\text { Autoimmune regulator, APECED } \\
\text { protein (O43918) }\end{array}$ \\
\hline KCNE1 (ISK) & $21 \mathrm{q} 22.12$ & $\begin{array}{c}\text { Potassium voltage-gated channel subfamily } \\
\text { E member } 1 \text { (P15382) }\end{array}$ & C21orf 2 (KNP-5;YFS/A2) & 21922.3 & Protein C21 orf $2(\mathrm{O} 43822)$ \\
\hline $\begin{array}{c}\text { RUNX1 } \\
\text { (AML-1;CBFA2;PEBP2 }\end{array}$ & $\begin{array}{l}21 \mathrm{q} 22.12 \\
2 ? \mathrm{~B})\end{array}$ & $\begin{array}{c}\text { Runt-related transcription factor 1 RUNX1 } \\
\text { or CBFA2 or AML1 (Q01196) }\end{array}$ & ITGB2 (CD18;MF17;LFA & \begin{tabular}{|l|l|}
$-1)$ \\
$21 q 22.3$ \\
\end{tabular} & $\begin{array}{l}\text { Integrin beta-2 precursor, CD18 } \\
\text { (P05107) }\end{array}$ \\
\hline CBR1 (CBR;CBR-1;CRN) & $21 \mathrm{q} 22.13$ & Carbonyl reductase [NADPH] 1 (P16152) & ADARB1 (hADAR2; RED & $\begin{array}{l}\text { D1) } \\
21 \mathrm{q} 22.3\end{array}$ & $\begin{array}{c}\text { Double-standed RNA } \\
\text { specific editase } 1 \text { (P78563) }\end{array}$ \\
\hline CHAF1B (CAF1A, CAF1A & $\begin{array}{r}\text { A-LSB) } \\
21 \mathrm{q} 22.13\end{array}$ & $\begin{array}{l}\text { Chromatin assembly factor } 1 \text { subunit B } \\
\text { (Q13112) }\end{array}$ & SLC19A1 (FOLT1; RFC1) & 21922.3 & $\begin{array}{c}\text { Human reduced folate carrier (RFC) } \\
\text { (P41440) }\end{array}$ \\
\hline SIM2 (HSSIM2) & $21 \mathrm{q} 22.13$ & Single-minded homolog 2 (Q14190) & COL6A1 & $21 \mathrm{q} 22.3$ & $\begin{array}{l}\text { Collagen alpha } 1(\mathrm{VI}) \text { chain } \\
\text { [Precursor] }(\mathrm{P} 12109)\end{array}$ \\
\hline HLCS (MCD) & $21 \mathrm{q} 22.13$ & $\begin{array}{c}\text { Biotin-protein ligase, } \\
\text { Holocarboxylase synthetase (P50747) }\end{array}$ & FTCD & $21 \mathrm{q} 22.3$ & $\begin{array}{l}\text { Formimidoyltransferase- } \\
\text { cyclodeaminase (O95954) }\end{array}$ \\
\hline DSCR6 & $21 \mathrm{q} 22.13$ & $\begin{array}{c}\text { Down Syndrome critical region protein } 6 \\
\text { (P57055) }\end{array}$ & PCNT2 (PCNT) & 21922.3 & Pericentrin $2(095613)$ \\
\hline DSCR5 (DCRC) & $21 \mathrm{q} 22.13$ & $\begin{array}{c}\text { Down Syndrome critical region protein } 5 \\
\text { (P57054) }\end{array}$ & S100B (S-100;RNR4) & $21 \mathrm{q} 22.3$ & S-100 protein beta chain ( $\mathrm{P} 04271)$ \\
\hline
\end{tabular}

Fig. 1. Human fetal brain protein expression of genes encoded on chromosome 21. 
The major finding of increased transcription regulator BACH1 may form the link to previous work pointing to and explaining a role for oxidative stress in DS brain as early as in the second trimester of pregnancy. BACH1 represses the expression of the antioxidant enzyme heme oxygenase-1, and indeed oxidative stress has been reported in DS neurons as well as in fetal DS brain [5], a mechanism that may well explain the development of DS neuropathology, including Alzheimer's disease-related neuropathology in DS brain, which inevitably supervenes from the fourth decade. Chromosome 21 open reading frame 2 protein and ES1 protein homolog mitochondrial precursor were elevated as well, probably reflecting or contributing to the mitochondrial defect known to occur in DS, which in turn may be linked to oxidative stress. The possible significance of interferon-alpha/beta recep- tor beta chain overexpression in terms of a pathogenetic role in DS remains open.

The only protein that was manifold reduced was SAM domain protein SAMSN-1 (HACS1). This structure contains an $\mathrm{SH} 3$ domain, and derangement may point to abnormal SH3-mediated signaling; such abnormal signaling has already been reported in fetal DS [6].

We are now in the process of performing functional in vitro and in vivo studies based upon dysregulation of these 9 proteins out of the 56 proteins whose genes are encoded on chromosome 21 . These studies will test the relevance of protein derangement for the development of the brain phenotype and pathogenesis of abnormal brain development in DS. We propose that the gene dosage hypothesis may apply for some gene products but should not be considered a general principle (fig. 1).

\section{References}

Aberrant Chromosome 21 Gene Products
1 Engidawork E, Lubec G: Molecular changes in fetal Down syndrome brain. J Neurochem 2003;84:895-904.

2 Engidawork E, Gulesserian T, Fountoulakis M, Lubec G: Aberrant protein expression in cerebral cortex of fetus with Down syndrome. Neuroscience 2003;122:145-154.

3 Yoo BC, Lubec G: p25 protein in neurodegeneration. Nature 2001;411:763-764.

4 Hine RJ, James SJ: Down syndrome and folic acid update. J Am Diet Assoc 2000;100:1004.
5 Busciglio J, Yankner BA: Apoptosis and increased generation of reactive oxygen species in Down's syndrome neurons in vitro. Nature 1995;378:776-779.

6 Peyrl A, Weitzdoerfer R, Gulesserian T, Fountoulakis M, Lubec G: Aberrant expression of signaling-related proteins 14-3-3 gamma and RACK1 in fetal Down syndrome brain (trisomy 21). Electrophoresis 2002;23:152-157. 